\title{
Elastoplasticity of auxetic materials
}

\author{
J. Dirrenberger ${ }^{\mathrm{a}, *}$, S. Forest ${ }^{\mathrm{a}}$, D. Jeulin ${ }^{\mathrm{a}, \mathrm{b}}$ \\ a Centre des Matériaux, MINES-ParisTech, CNRS UMR 7633, BP 87, 91003 Evry Cedex, France \\ ${ }^{\mathrm{b}}$ Centre de Morphologie Mathématique, MINES-ParisTech, 35, rue St-Honoré, 77305 Fontainebleau, France
}

\section{A R T I C L E I N F O}

Article history:

Received 15 October 2011

Received in revised form 13 March 2012

Accepted 15 March 2012

Available online 9 April 2012

\section{Keywords:}

Homogenization

Anisotropic compressible plasticity

Auxetics

Negative Poisson's ratio

Architectured materials

Finite element method

Periodic boundary conditions

\begin{abstract}
A B S T R A C T
Materials exhibiting a negative Poisson's ratio (auxetics) have drawn attention for the past two decades, especially in the field of lightweight composite structures and cellular media. Studies have shown that auxeticity may result in higher shear modulus, indentation toughness and acoustic damping. Although elastic properties of such materials have been extensively investigated, the effect of plasticity on auxetic behavior has not been discussed. In particular, does the auxetic character of the material remain while entering the plastic domain? The present work aims at modeling the nonlinear mechanical response of auxetics. Full-field simulations are performed using the finite element method with periodic boundary conditions. Macroscopic modeling of auxetics is attempted using an anisotropic compressible plasticity framework.
\end{abstract}

(c) 2012 Elsevier B.V. All rights reserved.

\section{Introduction}

In the case of isotropic elasticity, mechanical behavior can be described by 2 parameters only, Young's modulus $E$ and Poisson's ratio $v$ for instance. Poisson's ratio is defined in tension as the ratio of the contraction in the transverse direction to the extension in the longitudinal direction. Thermodynamically, $v$ lies between -1 and 0.5 . Most materials naturally present a positive Poisson's ratio, although negative Poisson's ratio materials, or auxetics [1], have been studied for more than two decades [2-8]. Such materials are expected to exhibit enhanced mechanical properties such as shear modulus and fracture toughness [9], indentation resistance [10-12] but also acoustic damping $[13,14]$ and crash resistance [15]. Moreover, $v<0$ allows synclastic curvature of plates [16], thus enabling the manufacture of doubly-curved sandwich panels without core buckling. To our knowledge the effect of plasticity on auxetic behavior has not been assessed from a computational mechanics perspective yet, this is the purpose of the present paper.

The microstructure considered and homogenization of the elastic properties are presented in Section 2. An extension to elastoplasticity based on full-field simulations is performed in Section 3 using finite elements (FEs) calculations with periodic boundary conditions. In Section 4, an isotropic compressible plasticity model is presented in order to define a plastic Poisson's ratio. An anisotropic compressible plasticity framework is then introduced for modeling

\footnotetext{
* Corresponding author. Tel.: +33 160763069; fax: +33 160763150.

E-mail address: justin.dirrenberger@mines-paristech.fr (J. Dirrenberger).
}

the homogenized nonlinear behavior of the auxetic lattice considered. Results of identification for model parameters obtained from FE simulations and optimization based on a Nelder-Mead algorithm are presented in Section 5.

\section{Homogenization of an elastic auxetic microstructure}

In this work, numerical homogenization is first used for determining the effective elastic properties of the materials considered. Computation is done over a unit-cell (defined by its periodicity vectors $\underline{\boldsymbol{v}}_{\boldsymbol{i}}$ ) with periodic boundary conditions (PBC) using $\mathrm{FE}^{1}$. The macroscopic stress and strain tensors $\boldsymbol{\Sigma}$ and $\boldsymbol{E}$ are defined by the spatial averages:

$\underset{\sim}{\boldsymbol{\Sigma}} \hat{=}\langle\underset{\sim}{\boldsymbol{\sigma}}\rangle=\frac{1}{V} \int_{V} \underset{\sim}{\boldsymbol{\sigma}} d V, \quad \underset{\sim}{\boldsymbol{E}} \hat{=}\langle\underset{\sim}{\boldsymbol{\varepsilon}}\rangle=\frac{1}{V} \int_{V} \underset{\sim}{\boldsymbol{\varepsilon}} d V$

PBC over the unit cell give displacement field $\underline{\boldsymbol{u}}$ such as:

$\underline{\boldsymbol{u}}=\underline{\boldsymbol{E}} \cdot \underline{\boldsymbol{x}}+\underline{\boldsymbol{v}} \quad \forall \underline{\boldsymbol{x}} \in V$

with $\underline{\boldsymbol{x}}$, the material point location vector and $\underline{\boldsymbol{v}}$, the periodic fluctuation. $\underline{v}$ takes the same value at two homologous points on opposite faces of $V$, whereas the traction vector $\underline{\boldsymbol{t}}=\boldsymbol{\sigma} \cdot \underline{\boldsymbol{n}}$ takes opposite values, $\underline{\boldsymbol{n}}$ being the normal vector.

\footnotetext{
${ }^{1}$ Z-set code: www.zset-software.com.
} 
By applying either macroscopic strain or stress, one can compute the effective fourth-rank tensors of elastic moduli $\boldsymbol{C}$ and compliances $\boldsymbol{S}$ defined such as:

$\underset{\sim}{\boldsymbol{\Sigma}}=\underset{\approx}{\boldsymbol{C}}: \underset{\sim}{\boldsymbol{E}}, \underset{\sim}{\boldsymbol{E}}=\underset{\approx}{\boldsymbol{S}}: \underset{\sim}{\boldsymbol{\Sigma}}$

For the purpose of this work, normalized Young's modulus, shear modulus and effective Poisson's ratio are defined in plane $(1,2)$ as follows:

$E=\frac{1}{E_{0} f_{V}} \frac{\Sigma_{11}}{\mathbf{E}_{11}} ; \quad \mu=\frac{1}{\mu_{0} f_{V}} \frac{\Sigma_{12}}{\mathbf{E}_{12}} ; \quad v^{\mathrm{eff}}=-\frac{\mathbf{E}_{22}}{\mathbf{E}_{11}}$

with local constitutive parameters $E_{0}$ (Young's modulus) and $\mu_{0}$ (shear modulus).

\subsection{Hexachiral lattice}

The medium studied in this work is an auxetic periodic lattice, it is considered for being used as core material for sandwich panels. Ref. [8] deals with the characterization of anisotropic elastic properties for this microstructure. Volume fraction $f_{V}$ considered here is $15 \%$. This chiral microstructure was first proposed in [5] and studied in $[7,17,8]$. The 6 -fold symmetry provides transverse isotropy (cf. Fig. 1). Chirality is necessary for this lattice to be auxetic. The tensor of elastic moduli for this material is expressed in MPa using Voigt notation in Eq. (5). Local constitutive parameters are listed in Table 1. A similar kind of 6 -fold symmetric chiral lattice was proposed and studied in [18], yielding the same value for the effective Poisson's ratio: $v^{\text {eff }}=-0.73$, as shown in Table 2 .

$\underset{\approx}{\boldsymbol{C}}]=\left[\begin{array}{cccccc}1650 & -1218 & 130 & 0 & 0 & 0 \\ -1218 & 1650 & 130 & 0 & 0 & 0 \\ 130 & 130 & 31968 & 0 & 0 & 0 \\ 0 & 0 & 0 & 5075 & 0 & 0 \\ 0 & 0 & 0 & 0 & 5075 & 0 \\ 0 & 0 & 0 & 0 & 0 & 1434\end{array}\right]$

The effective elastic properties presented in this part will be used for mechanical modeling in Section 4.

\section{Extension to elastoplasticity}

Let us consider the following yield function $f \underset{\sim}{\boldsymbol{\sigma}})$ :

$f(\underset{\sim}{\boldsymbol{\sigma}})=\sigma^{e q}-r$

with the von Mises equivalent stress,

$\sigma^{e q}=\sqrt{\frac{3}{2} \underset{\sim}{\boldsymbol{\sigma}^{d e v}}: \boldsymbol{\sigma}_{\sim}^{d e v}}$

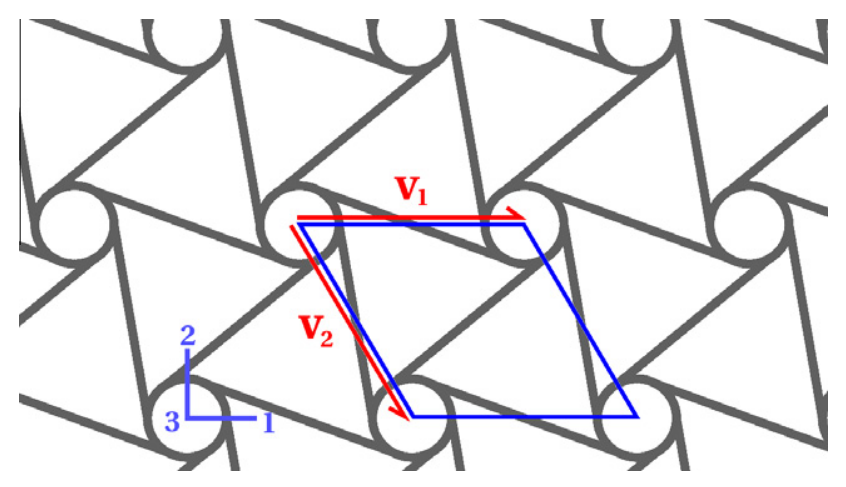

Fig. 1. Hexachiral unit-cell and periodicity vectors $\underline{\underline{v}}_{1}$ and $\underline{\mathbf{v}}_{2}$.
Table 1

Local constitutive elastic parameters.

$\begin{array}{ll}\text { Young's modulus (GPa) } & 210 \\ \text { Poisson's ratio } & 0.3\end{array}$

Table 2

Effective in-plane elastic properties of the hexachiral lattice.

\begin{tabular}{lc}
\hline & Hexachiral \\
\hline Normalized Young's modulus & 0.0235 \\
Normalized shear modulus & 0.2339 \\
Effective Poisson's ratio & -0.73 \\
\hline
\end{tabular}

where $\boldsymbol{\sigma}^{\text {dev }}$ is the deviatoric part of the stress tensor. Linear isotropic hardening rule is adopted:

$r=r_{0}+h p$

where $r_{0}$ is the yield stress, $h$ the hardening modulus and $p$ is the cumulative plastic strain variable.

Local material is now considered isotropic von Mises elastoplastic. Plastic material parameters are shown in Table 3.

First, the auxetic behavior is investigated. Although the parameters given in Table 3 will be used in the following sections, a short parametric study has been performed in order to assess the effect of the hardening modulus on the Poisson's ratio. Uniaxial straincontrolled tensile test is performed along direction 1 until $4 \%$ of total macroscopic strain. The homogenized cell exhibits a nonlinear elastoplastic behavior (cf. Fig. 2). Now, if one considers the ratio of transverse over longitudinal macroscopic strains, an apparent Poisson's ratio can be defined in the nonlinear regime as defined in Eq. (9) and plotted on Fig. 2. From these curves we observe that the auxetic nature of the lattice is kept with plasticity. The effect is even stronger than in elasticity when the hardening modulus is in the range ( $h=100 \mathrm{MPa}$ and $h=1000 \mathrm{MPa}$ ). The auxetic effect is dependent on the size of the plastic zone in the unit cell. If the plastic zone is confined in a small domain around the junction between the rotating nodes and the connecting beams, as shown on Fig. 3 for low values of the hardening modulus, the auxetic deformation mechanism is strengthened. For $h=10000 \mathrm{MPa}$, the plastic zone spreads almost over the entire cell, thus fading the effect of plasticity on the auxetic behavior. The hardening modulus value, $h=1000 \mathrm{MPa}$, is kept for the rest of this work since it is of the same order of magnitude of several common alloys.

$v^{\mathrm{app}}=-\frac{\mathbf{E}_{22}}{\mathbf{E}_{11}}$

Now, anisotropy in the plastic regime is investigated. As a matter of fact, there is no guarantee for the 6-fold symmetric material to behave isotropically in the plastic regime. Polar plots shown on Figs. 4-6 are obtained from uniaxial tensile and shear tests in every direction of the plane $(1,2)$. Each point corresponds to a test for a different direction with angle $\phi$ from the principal direction 1 of the structure defined on Fig. 3. Figs. 4 and 6 shows stress level vs. angle $\phi$ for three given strain states: respectively $0.2 \%$ (green), $1 \%$

Table 3

Local constitutive plastic parameters.

\begin{tabular}{lr} 
Yield stress (MPa) & 100 \\
Hardening modulus (MPa) & 1000 \\
\hline
\end{tabular}




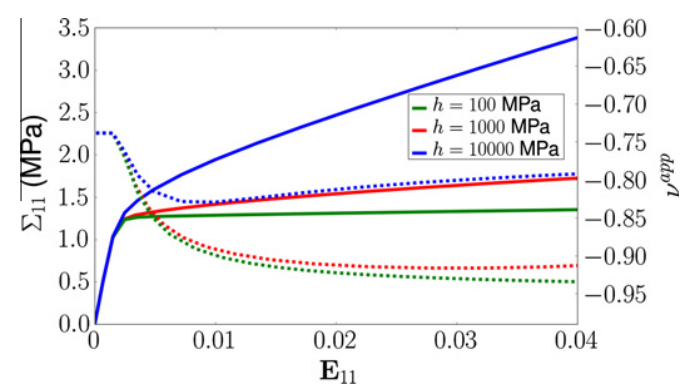

Fig. 2. Stress (plain curves) and apparent Poisson's ratio (dashed curves) vs. strain response for three different hardening moduli.

(red) and $4 \%$ (blue) total strain for tension, and $0.1 \%, 0.5 \%$ and $2 \%$ for shear. Fig. 5 uses the same color code but for the apparent Poisson's ratio vs. angle $\phi$ for the same given tension states. The three plots show a quasi-transversely isotropic response for the hexachiral lattice with plasticity.

\section{Macroscopic modeling}

An additional upscaling is performed. The mesoscopic elastoplastic behavior obtained in Section 3 is now modeled as a constitutive behavior for further use in large structural computations. First, let us consider an isotropic compressible plasticity model such as those developed by Green [19] and Abouaf et al. [20] for porous metals, and by Miller [21] and Deshpande and Fleck [22] for cellular materials. An extension to the anisotropic case was proposed by Badiche et al. [23] and Forest et al. [24].

Let us now consider a yield function $f(\boldsymbol{\Sigma})$ such as,

$f(\mathbf{\Sigma})=\Sigma_{e q}-R$

where $R$ is the macroscopic yield stress. Moreover, let us adopt the following equivalent yield stress:

$\Sigma_{e q}=\sqrt{\frac{3}{2} C_{\sim}^{\Sigma^{d e v}}: \underset{\sim}{\boldsymbol{\Sigma}^{d e v}}+F(\operatorname{Tr} \underset{\sim}{\boldsymbol{\Sigma}})^{2}}$

where $\operatorname{Tr} \boldsymbol{\Sigma}$ is the trace of the stress tensor. $C$ and $F$ are coefficients accounting for the relative influence of deviatoric and hydrostatic stress, they are usually expressed as functions of the porosity $\rho$ for isotropic materials.

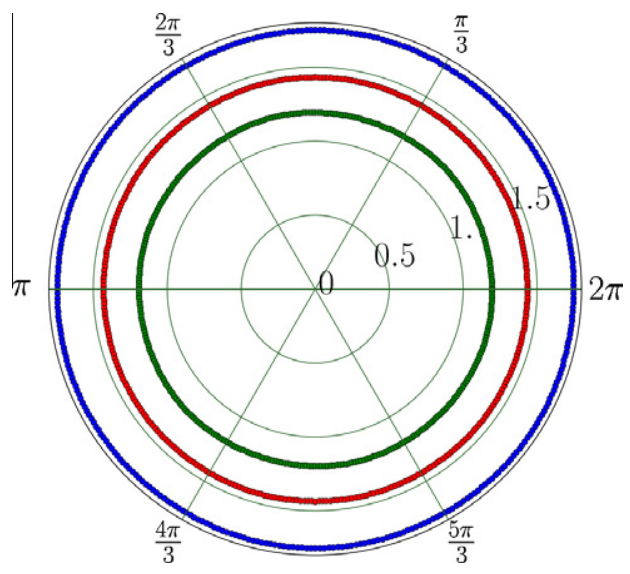

Fig. 4. Stress level (MPa) for $0.2 \%$ (green), $1 \%$ (red) and $4 \%$ (blue) total strain. (For interpretation of the references to color in this figure legend, the reader is referred to the web version of this article.)

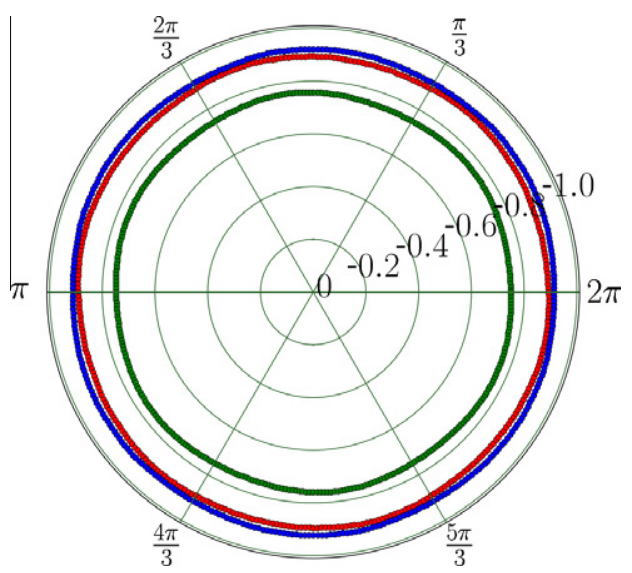

Fig. 5. Apparent Poisson's ratio for $0.2 \%$ (green), $1 \%$ (red) and $4 \%$ (blue) total strain. (For interpretation of the references to color in this figure legend, the reader is referred to the web version of this article.)

Associated plasticity is assumed, such as the macroscopic plastic strain rate is:

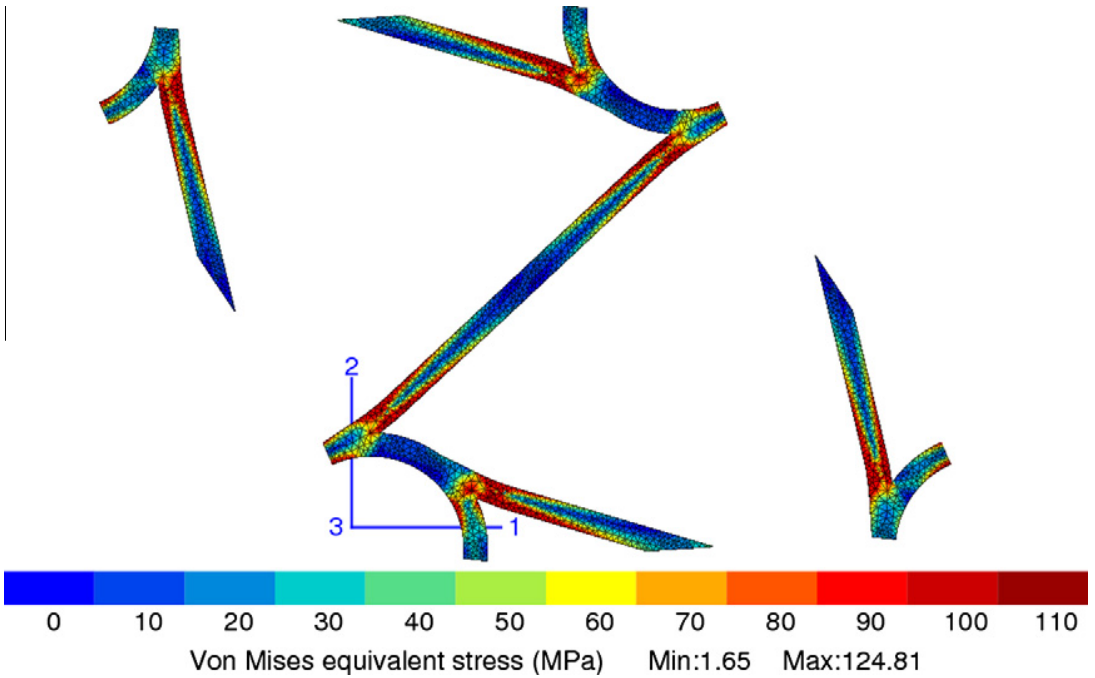

Fig. 3. Deformed shape of unit-cell after $4 \%$ of total strain, with von Mises equivalent stress map $(h=1000 \mathrm{MPa})$. 


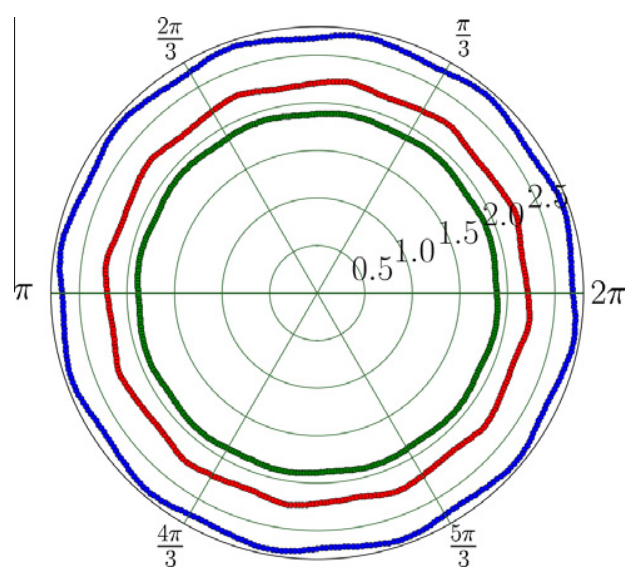

Fig. 6. Stress level (MPa) for $0.1 \%$ (green), $0.5 \%$ (red) and $2 \%$ (blue) total shear strain.

$\underset{\sim}{\dot{\mathbf{E}}^{p}}=\frac{\partial f}{\partial \boldsymbol{\Sigma}}=\frac{\dot{p}}{\sigma_{e q}}\left(\frac{3}{2} C \boldsymbol{\Sigma}^{d e v}+F(\operatorname{Tr} \underset{\sim}{\boldsymbol{\Sigma}}) \underset{\sim}{\mathbf{1}}\right)$

In the case of uniaxial tension, we define the in-plane plastic Poisson's ratio:

$v^{p}=-\frac{\dot{\mathbf{E}}_{22}^{p}}{\dot{\mathbf{E}}_{11}^{p}}=-\frac{F-\frac{C}{2}}{C+F}=\frac{\frac{C}{2}-F}{C+F}$

When $F=0$, incompressible plasticity is recovered. If $C=1$, then $v^{p}<0$ for $F>\frac{1}{2}$ and $\lim _{F \rightarrow+\infty} v^{p}=-1 . v^{p}$ as a function of $F$ is plotted on Fig. 7.

Such a plasticity model is not fully capable of describing the anisotropic behavior of our microstructure along direction 3, especially transverse contraction when tension is applied in plane (1, 2 ). In order to simplify the model, instead of using a fully anisotropic Hill tensor applied to the deviatoric stress tensor and a separate contribution of the hydrostatic stress, we consider here a generalized Hill tensor applied to the Cauchy stress tensor.

We consider the same yield function $f(\boldsymbol{\Sigma})$ as in Eq. (10) with the following equivalent yield stress:

$\Sigma^{e q}=\sqrt{\underset{\sim}{\boldsymbol{\Sigma}: \underset{\approx}{\boldsymbol{H}: \boldsymbol{\Sigma}}}}$

where $\boldsymbol{H}$ is the applied generalized Hill fourth-rank tensor.

For the hardening rule, we consider an isotropic hardening function with a nonlinear potential and a linear part:

$\mathrm{R}=\mathrm{R}_{0}+\mathrm{H} p+\mathrm{Q}\left(1-e^{-\mathrm{bp}}\right)$

\section{Simulation and identification}

In order to determine parameters for the model, we first estimate some of them from reference curves obtained by periodic

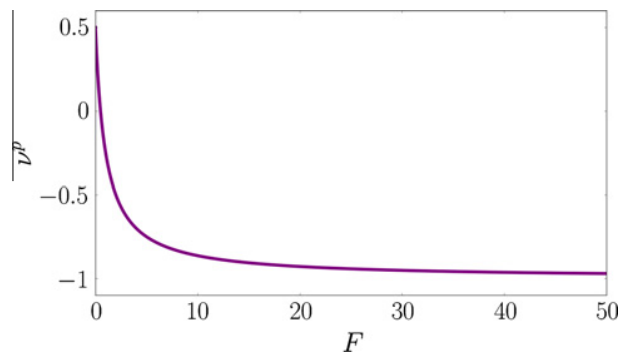

Fig. 7. Plastic Poisson's ratio for an isotropic material as a function of parameter $F$, with $C=1$. (For interpretation of the references to color in this figure legend, the reader is referred to the web version of this article.)

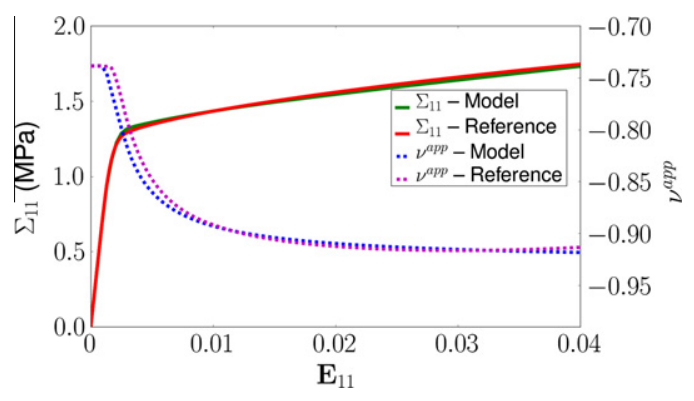

Fig. 8. Stress and apparent Poisson's ratio vs. strain for full-field simulation and macroscopic model for an uniaxial tensile test along direction 1.

simulations of the unit-cell. Then comparison between reference data with results computed on a RVE is made and optimization of macroscopic material parameters is run using a Nelder-Mead (simplex) algorithm. The experimental database includes tensile, shear and Poisson's ratio curves. While loading in tension, we consider out-of-plane contraction. However, we do not take into account tension in direction 3 and out-of-plane shear. Tensorial components of $\boldsymbol{H}$ (cf. Eq. (19)) and parameters for the hardening rule (15) are thus identified:

$R=1.29+8.61 p+0.1\left(1-e^{-140 p}\right)$

Identification of the hardening rule was also performed for the two other constitutive hardening moduli: $h=100 \mathrm{MPa}$ (Eq. (17)) and $h=10000 \mathrm{MPa}$ (Eq. (18)) in order to test the robustness of the identification scheme and to verify the effect of the local hardening modulus on the macroscopic hardening rule:

$R=1.26+0.62 p+0.1\left(1-e^{-160 p}\right)$

$R=1.28+25.9 p+1.07\left(1-e^{-86 p}\right)$

We observe that linear hardening modulus in the macroscopic rule increases with the local hardening modulus.

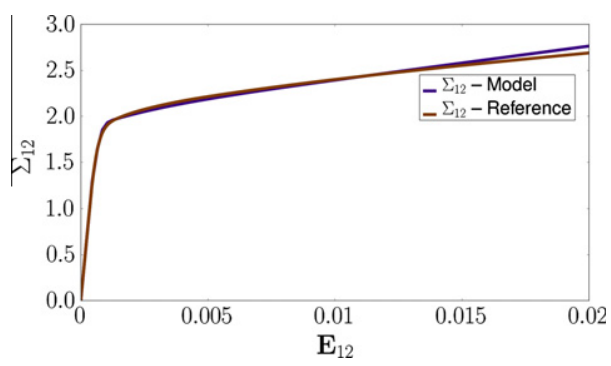

Fig. 9. Stress vs. strain for full-field simulation and macroscopic model for an pure shear test in plane $(1,2)$.

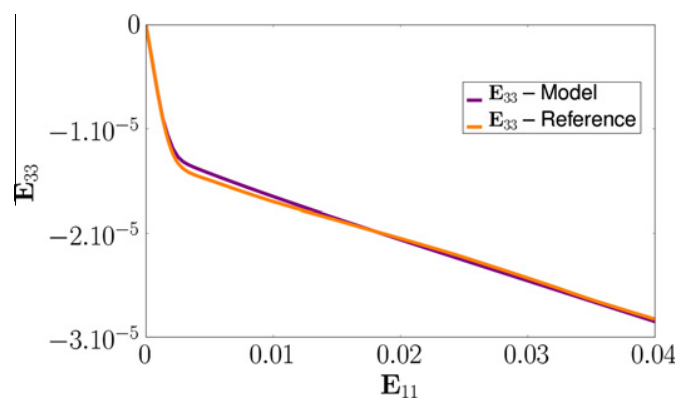

Fig. 10. Transverse strain vs. longitudinal strain for full-field simulation and macroscopic model for an uniaxial tensile test along direction 1 . 
Comparison between curves from full-field simulations and the identified macroscopic model provides a good correlation as shown on the tensile stress and apparent Poisson's ratio vs. strain curve (cf. Fig. 8), the shear stress vs. strain curve (cf. Fig. 9) and the transverse strain vs. longitudinal strain curve (cf. Fig. 10).

$$
\underset{\approx}{\boldsymbol{H}}]=\left[\begin{array}{cccccc}
1.00 & 0.9294 & -0.00031 & 0.0006 & 0 & 0 \\
0.9294 & 0.99 & -0.00027 & -0.00067 & 0 & 0 \\
-0.00031 & -0.00027 & \times & 0 & 0 & 0 \\
0.0006 & -0.00067 & 0 & 0.11554 & 0 & 0 \\
0 & 0 & 0 & 0 & \times & 0 \\
0 & 0 & 0 & 0 & 0 & \times
\end{array}\right]
$$

\section{Conclusions and prospects}

Full-field simulations and macroscopic modeling using an anisotropic compressible plasticity framework have been performed for an auxetic microstructure: the hexachiral lattice. Plasticity of auxetics has been explored, showing that the auxetic effect persists and becomes even stronger with plastic yielding. It was also shown that the effect of plasticity on auxeticity fades with the expansion of the plastic zone. The plastic response anisotropy for this 6-fold symmetric lattice is becoming weaker with plastic saturation. The proposed fully anisotropic Hill criterion seems to be suitable for modeling architectured cellular materials as it was able to catch negative Poisson's ratio, transverse contraction, and volume change. Further work will include the modeling of other auxetic microstructures, a parametric study of the influence of the yield stress and the local hardening rule on the homogenized plastic behavior and the simulation of an indentation test using the macroscopic model developed in this work.

\section{Acknowledgments}

This work is part of the MANSART (Architectured sandwich materials) Project ANR-08-MAPR-0026. Financial support of ANR is gratefully acknowledged.

\section{References}

[1] K.E. Evans, M.A. Nkansah, I.J. Hutchinson, S.C. Rogers, Nature 353 (1991) 124

[2] R.F. Almgren, Journal of Elasticity 15 (1985) 427-430.

[3] R.S. Lakes, Science 235 (1987) 1038-1040.

[4] B.D. Caddock, K.E. Evans, Journal of Physics D: Applied Physics 22 (1989) 18771882.

[5] R.S. Lakes, Journal of Materials Science 26 (1991) 2287-2292.

[6] G.W. Milton, Journal of the Mechanics and Physics of Solids 40 (5) (1992) 1105-1137.

[7] D. Prall, R.S. Lakes, International Journal of Mechanical Sciences 39 (3) (1997) 305-314.

[8] J. Dirrenberger, S. Forest, D. Jeulin, C. Colin, Procedia Engineering 10(2011) 18471852, http://dx.doi.org/10.1016/j.proeng.2011.04.307 (11th International Con ference on the Mechanical Behavior of Materials (ICM11)).

[9] J.B. Choi, R.S. Lakes, International Journal of Fracture 80 (1996) 73-83.

[10] R.S. Lakes, Nature 361 (1993) 511-515.

[11] K.L. Alderson, A.P. Pickles, P.J. Neale, K.E. Evans, Acta Metallurgica et Materialia 42 (7) (1994) 2261-2266.

[12] K.L. Alderson, A. Fitzgerald, K.E. Evans, Journal of Materials Science 35 (16) (2000) 4039-4047.

[13] A.W. Lipsett, A.I. Beltzer, Journal of the Acoustical Society of America 84 (6) (1988) 2179-2180.

[14] C.P. Chen, R.S. Lakes, Journal of Engineering Materials and Technology 118 (3) (1996) 285-288.

[15] F. Scarpa, J.R. Yates, L.G. Ciffo, S. Patsias, Proceedings of the Institution of Mechanical Engineers Part C-Journal of Mechanical Engineering Science 216 (12) (2002) 1153-1156.

[16] K.E. Evans, Composite Structures 17 (2) (1991) 95-111.

[17] A. Alderson, K.L. Alderson, D. Attard, K.E. Evans, R. Gatt, J.N. Grima, W. Miller, N. Ravirala, C.W. Smith, K. Zied, Composites Science and Technology 70 (7) (2010) 1042-1048.

[18] H. Mitschke, J. Schwerdtfeger, F. Schury, M. Stingl, C. Körner, R.F. Singer, V. Robins, K. Mecke, G.E. Schröder-Turk, Advanced Materials 23 (2011) 26692674.

[19] R.J. Green, International Journal of Mechanical Sciences 14 (1972) 215-224.

[20] M. Abouaf, J.-L. Chenot, G. Raisson, P. Bauduin, International Journal for Numerical Methods in Engineering 25 (1988) 191-212.

[21] R.E. Miller, International Journal of Mechanical Sciences 42 (2000) 729-754.

[22] V.S. Deshpande, N.A. Fleck, Journal of the Mechanics and Physics of Solids 48 (2000) 1253-1283.

[23] X. Badiche, S. Forest, T. Guibert, Y. Bienvenu, J.-D. Bartout, P. Ienny, M. Croset, H. Bernet, Materials Science and Engineering A289 (2000) 276-288.

[24] S. Forest, J.-S. Blazy, Y. Chastel, F. Moussy, Journal of Materials Science 40 (2005) 5903-5910. 